\title{
Epidemiology and Trend of Cancers in the Province of Kerman: Southeast of Iran
}

\author{
Naeimeh Keyghobadi ${ }^{1}$, Hosein Rafiemanesh ${ }^{2}$, Abdollah Mohammadian- \\ Hafshejani $^{3}$, Mostafa Enayatrad ${ }^{4}$, Hamid Salehiniya ${ }^{2,5 *}$
}

\begin{abstract}
Background: According to increase in elderly populations, and change in lifestyle and cancer-causing behavior, the global burden of cancer is increasing. For prevention and control of disease, knowledge of population statistics of cancers and their trends is essential. This study aimed to investigate the epidemiology and trends of cancer in the province of Kerman: southeast of Iran. Materials and Methods: This analytical and cross-sectional study was carried out based on cancer registry data at the Disease Management Center of the Health Ministry from 2004 to 2009 in the province of Kerman in Iran. Common cancers were defined as the number of reported cases and standardized incidence rates. To compute the annual percentage change (APC), joinpoint 4.1.1.1 software was applied. Results: Of 10,595 registered cases, $45.3 \%$ (4802 cases) were in women and $56.7 \%(5,793$ cases) occurred in men. The standardized incidence rates for both females and males were increasing during the six years studied. The most common cancers in both sexes during six years of studied were skin $(13.4 \%)$, breast $(9.35 \%)$, bladder $(7.8 \%)$, stomach $(7.45 \%)$, leukemia $(7.05 \%)$, colorectal $(5.57 \%)$, lung $(4.92 \%)$, trachea $(3.51 \%)$ and prostate $(2.48 \%)$. Conclusions: Our findings revealed that the cancer incidence is demonstrating increasing trends in both sexes in the province of Kerman. This may be because of changes in lifestyle, increasing exposure to risk factors for cancer and increase of life expectancy. If this is the case, increasing public awareness of cancer risk factors is a high priority, together with introduction of large-scale screening techniques.
\end{abstract}

Keywords: Cancer - epidemiology - time trends - Iran

Asian Pac J Cancer Prev, 16 (4), 1409-1413

\section{Introduction}

Spread of non-communicable chronic diseases, especially cancer, along with control of communicable diseases has become a public health concern in the world (Taheri et al., 2012). According to increasing elderly population, change in lifestyle and enhancing cancercausing behaviors such as increasing tobacco consumption, unhealthy diet, lack of physical activity and other factors, global burden of cancer is increasing (Atrkar-Roushan et al., 2013; Moradpour and Fatemi 2013). Cancer is the fifth leading cause of death worldwide(Atrkar-Roushan et al., 2013) so that 7.6 million people annually lose their lives due to cancer and $70 \%$ of those are related to middle and low income countries (Fateh and Emamian 2013). The number of 1.7 million deaths annually occurs in Europe and it accounts for $20 \%$ of all deaths. In Sweden, 26\% and $22 \%$ deaths from cancer take place in men and women, respectively(Abdoli et al., 2014). Currently, cancer is the second leading cause of death in developed countries and the third in developing countries (Zeinalzadeh et al., 2012).

Five most common cancers in the world include lung, stomach, liver, colon and breast (Fateh and Emamian 2013) of which lung and breast cancers are the most common in men and women, respectively, globally (Zeinalzadeh et al., 2012). About 30,000 deaths annually caused by cancer in Iran revealed that it is the third major cause of death after cardiovascular diseases and accidents and expected that the number ofdeaths from cancer will increase to 62,000 people by 2020 (Fateh and Emamian 2013).The incidence rate of cancer is different in various regions of Iran by the age composition of populations, lifestyles and behaviors of individuals so that studies performed in different regions of the country, like Yazd, Shah rood and Khuzestan, showed that age-specific incidence rates per100,000 population were 104.7, 95.4 and 66.9, respectively (Fateh and Emamian 2013; Amoori et al., 2014; Vakili et al., 2014). The diseases as cancer have especial place in the health system because of

${ }^{1}$ Department of Biostatistics and Epidemiology, Faculty of Health, Sahib Sadoughi University of Medical Sciences, Yazd, Iran, ${ }^{2}$ Department of Epidemiology and Biostatistics, School of Public Health, Tehran University of Medical Sciences, Tehran, Iran, ${ }^{3}$ Department of Epidemiology and Biostatistics, School of Public Health, Isfahan University of Medical Sciences, Isfahan, ${ }^{4}$ Department of Epidemiology and Biostatistics, School of Public Health, Shahid Beheshti University of Medical Sciences, ${ }^{5}$ Minimally Invasive Surgery Research Center, Iran University of Medical Sciences, Tehran, Iran*For correspondence: alesaleh70@yahoo.com 
irreparable complications and high costs of diagnosis and treatment (Rahimi and Heidari 2012). To apply methods of preventing and controlling disease,

knowing the statistics of cancer and its trend is effective. Considering that no study on the trend of cancers was done in the province of Kerman, in Iran, this study aimed to investigate common cancer epidemiology and trend of its changes in the province.

\section{Materials and Methods}

This was an analytical and cross-sectional study carried out based on cancer registry data and the disease management center of health ministry from 2004 to 2009 in the province of Kerman. Kerman Province is one of the 31 provinces of Iran. Kerman is in the southeast of Iran. In 2011 the population of the province was 2,938,988 (1482339 male, 1456649 female) in 786,400 households.

Data was obtained after removing duplicate codes according to the organ involved in cancer and coding by ICD-O. Standardized incidence rates were calculated based on the world standard population. Data was analyzed using STATA (version 12) software and simple linear regression for calculating the simple linear slope of the annual increase. To compute the annual percentage change (APC), Join point 4.1.1.1 software was applied. The significance level was considered as 0.05. Common cancers were defined as the number of reported cases and standardized incidence rates.

\section{Results}

In this study, 10,595 registered cases of cancer were obtained for 6 years(Goya 2008). Of the total cases registered, $45.32 \%$ (4802 cases) were women and $56.68 \%$

Table 1. Number of Cases and Age-standardized Incidence Rates (per 100,000) by Sex (2009-2004)

\begin{tabular}{lcccc}
\hline & \multicolumn{2}{c}{ Male } & \multicolumn{2}{c}{ Female } \\
\hline Year & Count & ASR & Count & ASR \\
2004 & 671 & 74.93 & 572 & 72.69 \\
2005 & 691 & 71.43 & 554 & 61.79 \\
2006 & 915 & 85.85 & 765 & 82.72 \\
2007 & 837 & 79.2 & 727 & 78.88 \\
2008 & 1070 & 104.22 & 851 & 91.94 \\
2009 & 1609 & 131.61 & 1333 & 128.26 \\
\hline
\end{tabular}

(5,793 cases) occurred in men. The sex ratio (male to female) for all cancers was 1.21. The highest number of cancer cases (2942) in both sexes was seen in 2009. The age standardized incidence rate (ASR) showed the trend of increase for both sexes so that the standardized incidence rate for females was 72.69 in 2004 and the rate was increased to 128.26 in 2009. Standardized incidence rates were 74.93 and 131.61 in 2004 and 2009, respectively (Table1).

Linear regression analysis showed there was significantly incremental linear slope (10.41) for agestandardized incidence rate in females $(\mathrm{p}=0.03)$. The linear regression was significant for age-standardized incidence rate for males with an increasing slope of 10.71 ( $\mathrm{p}=0.03$ ). Regression analysis on the log scale, the annual percentage change(APC) for women(12.1, CI: 2-23.1) and for men (7.11, CI:3.2-20.9) and significant and positive trend were seen (Figures 1,2).

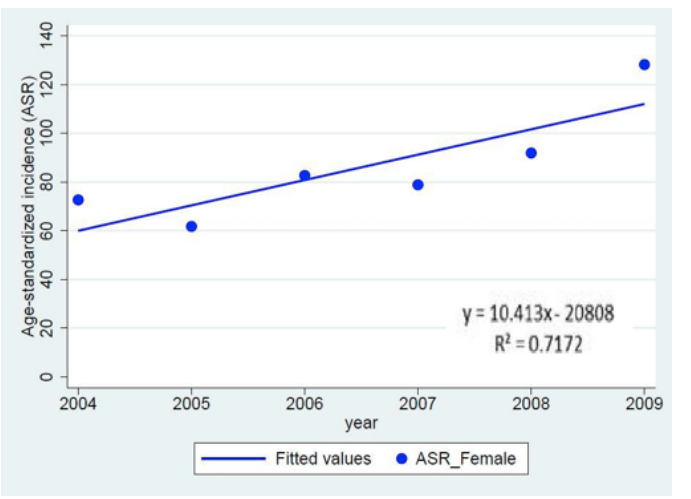

Figure 1. Trend of Cancer Incidence among Women in the Province of Kerman 2004-2009

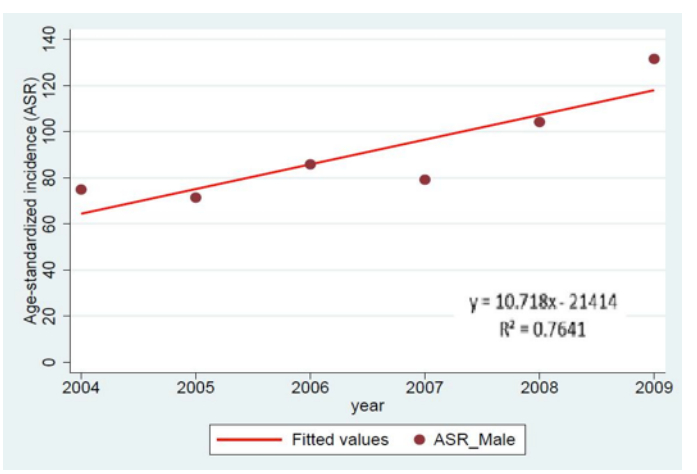

Figure 2. Trend of Cancer Incidence among Men in the Province of Kerman 2004-2009

Table 2. The Age-Standardized Incidence Rate (per 100,000) for Prevalent Cancers in Men and Women, Kerman, 2004-2009

\begin{tabular}{|c|c|c|c|c|c|c|c|}
\hline Cancer type & & 2004 & 2005 & 2006 & 2007 & 2008 & 2009 \\
\hline \multicolumn{8}{|l|}{ Women } \\
\hline 1. & Breast & 14.68 & 12.59 & 18.03 & 15.77 & 16.40 & 23.01 \\
\hline 2. & Skin & 10.73 & 7.32 & 11.98 & 13.80 & 14.62 & 13.23 \\
\hline 3. & Colon \& Rectum & 5.43 & 4.34 & 5.47 & 5.11 & 4.83 & 8.58 \\
\hline 4. & Leukemia & 3.45 & 3.73 & 5.34 & 4.61 & 3.67 & 7.30 \\
\hline 5. & Thyroid gland & 2.89 & 3.28 & 3.61 & 3.77 & 4.61 & 5.50 \\
\hline \multicolumn{8}{|l|}{ Men } \\
\hline 1. & Skin & 11.63 & 10.85 & 13.45 & 13.07 & 15.73 & 14.53 \\
\hline 2. & Bladder & 7.73 & 9.71 & 9.41 & 11.17 & 13.20 & 14.66 \\
\hline 3. & Stomach & 9.13 & 7.23 & 8.16 & 6.65 & 10.09 & 11.19 \\
\hline 4. & Leukemia & 5.53 & 4.55 & 8.60 & 7.84 & 6.22 & 8.74 \\
\hline 5. & Lung and Bronchus & 5.85 & 4.47 & 3.93 & 5.35 & 7.92 & 10.11 \\
\hline
\end{tabular}




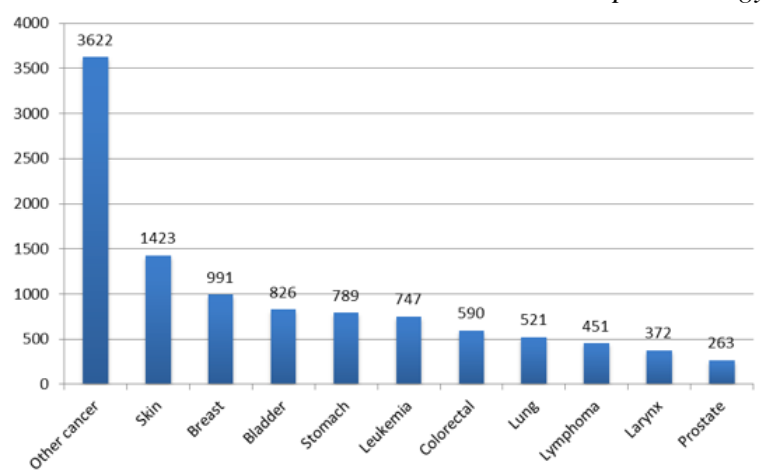

Figure 3. The Frequency of 10 Common Cancers, Kerman, 2004-2009

The most common cancers in both sexes during six years of studied were as follows: skin $(13.43 \%)$, breast $(9.35 \%)$, bladder $(7.8 \%)$, stomach $(7.45 \%)$, leukemia $(7.05 \%)$, colorectal $(5.57 \%)$, lung $(4.92 \%)$, trachea $(3.51 \%)$ and prostate $(2.48 \%)$, respectively (Figure 3$)$. Considering the frequency of disease, the five most common cancer were breast(963 cases), skin (611 cases), colorectal (291), leukemia (271 cases) and thyroid (262 cases) in women during 6 years, respectively, but in men skin (812 cases), bladder (663 cases), stomach (534 cases), leukemia (476 cases) and lung cancers (384 cases), respectively. The five cancers constituted $49.94 \%$ and $49.52 \%$ in men and women, respectively. The agestandardized incidence rate showed increasing trend for common cancers in men and women (Table 2).

\section{Discussion}

This study investigated the incidence of cancers and trend of its changes in the province. Cancer is one the most important causes of death in the world. It is estimatedthat $45 \%$ of cancer cases occur in developing countries by 2025 (Amoori et al., 2014). Of 74,067cases of cancer, $55.58 \%$ were in men and $41 / 44 \%$ in women in 2004 (Eatemed et al., 2008). In our study, sex ratio was 1.21.In other words, cancer cases were more in men than women and other studies also reported that the incidence of cancer was prevalent in men than women (Nejad et al., 2009; Roshandel et al., 2012; Amoori et al., 2014). Women are more sensitive than men to take care of their health, which can cause lower incidence of the disease, especially cancer, in females than males (Rahimi and Heidari, 2012). In US, of 2437163 registered deaths, 567,628 cases suffered from cancer in 2009. Cancer includes $23 \%$ of all cancer deaths and the second leading cause of death after cardiovascular diseases in US, while considered the third cause of death in Iran(Siegel et al., 2013). In the present study, the age-standardized incidence rate showed significant increase trend since 2004 to 2009. A study conducted in other regions in Iran indicated that the age standardized incidence has increased during 2004 to 2009 (Fateh and Emamian 2013; Amoori et al., 2014). In US, a study stated that cancer incidence rates for men and women were 550.7 and 419.3 per hundred thousand people, respectively, from 2004 to 2009 and the incidence of cancer has increasing trending both sexes during four years (Siegel et al., 2013). Our study expressed that percent of annual increase was 12.1 and 11.7 in women and men, respectively. Another study in a region of Iran, Shah rood city, cancer had percent of annual increase of 12.4 (Fateh and Emamian 2013). The incidence of cancer has also increased 2.5 times in males and 2.85 in females over five years in the province of Khuzestan of Iran (Amoori et al., 2014).

Skin $(12.3 \%)$, breast $(10.6 \%)$, stomach $(9.3 \%)$, colorectal $(8.4 \%)$, bladder $(6.2 \%)$ and prostate $(5.2 \%)$ cancers were the most common cancers in both sexes in Iran (Eatemed et al., 2008). This study determined that skin, breast, bladder, stomach, leukemia, colorectal, lung, lymphoma, trachea and prostate cancers are ten common cancers in both sexes, respectively.

According to the national cancer registry, cancer is the first rank in Iranian men and the second rank in Iranian women (Eatemed et al., 2008). In our study, skin cancer was the first rank in both sexes, the first in men and the second in women. As well, the age-standardized incidence rates related to this cancer were 0.7 and 0.6 in men and women, respectively, while the rate for Iranian men and women were 18.93 and 13.09, respectively (Eatemed et al., 2008) and in the present study 14.53 and 13.23 in men and women, respectively, which were greater than the global statistics. The most important risk factor for skin cancer is sun radiation. Ultraviolet waves and radiation from the sun in Iran, especially in central and southern parts of the country, are more so that lead to the increase of the cancer in this regions. This study also showed that incidence of skin cancer in men and women was changeable so that the incidence rate of skin cancer in 2007 was lower in 2008. This may be due to increase awareness about the importance of protecting your skin against sun radiation. In other regions of the country (such as the provinces of Yazd and Markazi, skin cancer was the most common type of cancer in both sexes (Mohafhegh et al., 2008; Amoori et al. 2014; Vakili et al., 2014), but the cancer was the second most common cancer in both sexes in other areas (the city of Shah rood and the province of eastern Azerbaijan (Fateh and Emamian, 2013; Mokarian et al., 2011).

Breast cancer is known as the most common malignancy in women because its age-specific incidence rate was 38.9 in the world and it encompasses a quarter of all cancers (Pimhanam et al., 2014). The cancer is considered the first rank because of theage-specificincidenceof 28.25 (Eatemed et al., 2008). In the present study, the agespecific incidence rate of 23.01 for this cancer in 2008 indicated that it is the most common cancer in women and its trend is increasing during 6 years of study. Studies conducted in other areas (the provinces of Yazd, Khuzestan and Mazandaran) confirmed that breast cancer is also the most common cancer in women (Nejad et al., 2009; Amoori et al., 2014; Vakili et al., 2014). The incidence rate of breast cancer in young women in Asian countries is moderate but it has the increasing trend (Afsharfard et al., 2013). Hormone changes in women, enhancing the age of first child birth and breast feeding, an in appropriate lifestyle, such as high-fat diet and obesity, are risk factors of the cancer, which in developing countries like Iran there is an increasing trend of the risk factors and breast cancer (Moradpour and Fatemi, 2013). 
Bladder cancer with the age-specific incidence rate of 12.59 is the fourth most common cancer in Iranian men and with the age-specific incidence rate of 3.28 is the ninth most common cancer in Iranian women. The age-specific incidence rates of the cancer in developed, developing and the whole of world were 16.6, 5.4 and 10.8, respectively (Eatemed et al., 2008). It is the ninth most common cancer in the world. The highest incidence rate of the cancer was seen in North America and North Africa and most of deaths were reported from Egypt (Farahmand and Almasi-Hashiani, 2013). In our study, bladder cancer is the second most common cancer in men and has increased during 6 years of the study. Another study performed in the province of Fars (of the country), also showed that incidence of the cancer has increasing trend during 2003-2004 so that incidence ofbladdercancerfrom 6.9 perhundred thousandpopulationin 2002 reachedto 10.3 per hundred thousand population in 2007. Smoking, exposure to toxins and heavy metals are the most important risk factors for this cancer (Farahmand and Almasi-Hashiani 2013). Results of a national survey has shown that mean cigarette consumption was in Kerman (a central part of Iran) more than mean national consumption(Mehdi et al., 2011). As a result, one of the reasons for the high incidence of bladder cancer in this city can be caused by high cigarette consumption.

Stomach cancer one of the most important cancers of the digestive system. It is the third most common cancer in both sexes, the fourth most common cancer in women and the second most common cancer in men(Eatemed et al., 2008). The highest incidence of the cancer was observed in Eastern Asia, Western Europe and South America but the lowest rate was seen in North America and Africa (Jemal et al., 2011). In the country (Iran), the highest incidence rate of the cancer was seen in northern and north western provinces, the lowest incidence rate in the south and the moderate incidence in central and western provinces (Kolahdoozan et al., 2010). Ardabil (a province of north western Iran) had the highest incidence of stomach cancer because of age-specific incidence rates of 28.86 for both sexes and 63.76 for men in the country (Eatemed et al., 2008). The province of Semnan, Golestan, East Azerbaijan and Tehran were areas with high incidence of the cancer (Kolahdoozan et al., 2010). Against, the province of Kerman, located in the south of Iran, is considered a low-risk area for the cancer according to the age-specific incidence rate of 11.9 for men in 2009 butthe 6-year trend of it is increasing. In our study, stomach cancer was the fourth most common cancer in both sexes and the third most common cancer in men but not among the five most common cancers in women. Other studies also determined that it is not one of the five most common cancers in women but first and second most common cancer in men in some areas like the city of Shah rood and the province of Khuzestan, respectively (Fateh and Emamian. 2013; Amoori et al., 2014).

Leukemia cancer is the seventh most common cancer in the country. It is the sixth and seventh most common cancer in men and women, respectively, considering the age-specific incidence rates of 6.42 and 4.84 in men and women, respectively (Eatemed et al., 2008). In the present study Leukemia cancer is the fifth in both sexes and the fourth most common cancer in men and women. In the provinces of Yazd and Khuzestan, it is the fourth most common cancer in women (Amoori et al., 2014; Vakili et al., 2014). The cancer has changed from 2004 to 2008. In other words, it had increasing trend. In the country, the cities of Yazd, Ahvaz, Isfahan and Kerman had the highest incidence of leukemia (Eatemed et al, 2008). Smoking is one of the most important risk factor for the cancer. The results of a national study of US

showed that deaths from leukemia has reduced in countries where cigarette consumption has reduced but in countries where the prevalence of cigarette consumption has been constant, cancer incidence has remained unchanged (Fazeli et al., 2013). Given that one of the reasons for the increase of leukemia in this province (Kerman) is high cigarette smoking.

According to national reports in 2008, most incidence cases were seen in the province of Yazd for women and in Markazi for men. In Kerman, ninth and tenth ranks of cancer are related to women and men, respectively.

In conclusion, our findings revealed that the cancer incidence had increasing trend in both sexes in the province of kerman. that may be cause by changes in lifestyle, increasing exposure to risk factors for cancer, and the increase of life expectancy. therefore, cancer incidence is preventable by large-scale screening techniques and increasing public awareness of cancer risk factors. it is also suggested that similar studies performed in other areas of the country to assess trend of cancer and better understand the causes of the increase in cancer.

\section{References}

Abdoli G, Bottai M, Moradi T (2014). Cancer mortality by country of birth, sex and socioeconomic position in Sweden, 1961-2009. PloS one, 9, 93174.

Afsharfard A, Mozaffar M, Orang E, Tahmasbpour E (2013). Trends in epidemiology, clinical and histopathological characteristics of breast cancer in Iran: results of a 17 year study. Asian Pac J Cancer Prev, 14, 6905-11.

Amoori N, Mirzaei M, Cheraghi M (2014). Incidence of cancers in Kuzestan province of Iran: Trend from 2004 to 2008. Asian Pac J Cancer Prev, 15, 8345-9.

Atrkar-Roushan Z, Kazemnejad A, Mansour-Ghanaei F, et al (2013) Trend analysis of gastrointestinal cancer Incidences in Guilan province: comparing rates over 15 years. Asian Pac J Cancer Prev, 14, 7587-93.

Eatemed K, Gooya MM, Ramazani R, et al (2008). Iranian Annual National Cancer Registration Report. Ministry of Health and Medical Education, Center for Disease Control and Prevention (In Persian).

Farahmand M, Almasi-Hashiani A (2013). Epidemiology of bladder cancers in fars province, Southern Iran (2003-2008). J Kerman Univ Medical Sciences, 20, 387-394.

Fateh, Mansooreh, Mohammad Hassan Emamian (2013). Cancer incidence and trend analysis in Shahroud, Iran, 2000-2010. Iranian J Cancer Prev, 6, 85-94.

Fazeli, Pourhoseingholi MA, Vahedi M, et al (2013). Leukemia cancer mortality trend in Iran, from 1995 to 2004. Iranian J Cancer Prev, 6, 170-73.

Jemal A, Bray F, Center MM, et al (2011). Global cancer statistics. CA: Cancer J Clin, 61, 69-90. 
Kolahdoozan S, Sadjadi A, Radmard A, et al (2010). Five common cancers in Iran. Arch Iran Med, 13, 143-146.

Mehdi E, Vahdaninia M, Azin A, et al (2011). Survey of Iranian people about the prevalence of smoking using. Payesh $J$, 10, 365-72.

Mohafhegh F, Hamta A, Shariatzadeh SMA (2008). The study of cancer incidence and cancer registration in Markazi province between 2001-2006 and comparison with national statistics, Iran. Arak Medical University J, 11, 84-93.

Mokarian F, Ramezani MA, Heydari K, Tabatabaeian M, Tavazohi H (2011). Epidemiology and trend of cancer in Isfahan 2005-2010. J Res Med Sci, 16, 1228-33.

Moradpour F, Fatemi Z (2013). Estimation of the projections of the incidence rates, mortality and prevalence due to common cancer site in Isfahan, Iran. Asian Pacific J Cancer Prev, 14, 3581-5.

Nejad FN, Ramezani Daryasari R, Ghafari F (2009). Epidemiology of cancer in Mazandaran province 2006. $J$ Mazandaran Univ Medical Sciences, 19, 61-65.

Rahimi F, Heidari M (2012). Time trend analysis of stomach cancer incidence in the West of Iran. J Health Dev, 1, 100-11.

Roshandel G, Sadjadi A, Aarabi M, et al (2012). Cancer incidence in Golestan province: report of an ongoing population-based cancer registry in Iran between 2004 and 2008. Arch Iran Med, 15, 196-200.

Siegel R, Naishadham D, Jemal A (2013). Cancer statistics, 2013. CA: Cancer J Clin, 63, 11-30.

Taheri NS, Bakhshandehnosrat S, Tabiei M, et al (2012). Epidemiological pattern of breast cancer in Iranian women: is there an ethnic disparity. Asian Pac J Cancer Prev, 13, 4517-20.

Vakili M, Pirdehghan A, Adimi M, Sadeghian M, Akhondi M (2014). Epidemiology and trend of cancer in yazd, a central province of Iran, 2005-2009. J Res Hlth Sci, 14, 210-3.

Zeinalzadeh AH, et al (2012). Pattern of age distribution of different cancers in East Azerbaijan province, Iran. J Kerman Univ Medical Sciences, 19, 308-386. 